\title{
Perfil farmacoterapêutico de usuários de práticas integrativas e complementares
}

\author{
Fernanda Rocha Anjos de Oliveira \\ Estudante do Curso de Enfermagem da Universidade Federal de \\ Mato Grosso (UFMT), Campus de Rondonópolis/MT, Brasil \\ $\triangle$ fernandarochaanjos@gmail.com \\ Graziele Ferreira Pinto \\ Estudante do Curso de Enfermagem da Universidade Federal de \\ Mato Grosso (UFMT), Campus de Rondonópolis/MT, Brasil \\ Raquel Aparecida Nicácio \\ Estudante do Curso de Enfermagem da Universidade Federal de \\ Mato Grosso (UFMT), Campus de Rondonópolis/MT, Brasil \\ Renata Aparecida Faria de Araújo \\ Estudante do Curso de Enfermagem da Universidade Federal de \\ Mato Grosso (UFMT), Campus de Rondonópolis/MT, Brasil \\ Débora Aparecida da Silva Santos \\ Doutora em Recursos Naturais (Saúde e Meio Ambiente) \\ pela Universidade Federal de Campina Grande (UFCG)

\section{Magda de Mattos} \\ Doutora em em Educação pelo Instituto de Educação da Universidade Federal de Mato Grosso (UFMT)

\section{Letícia Silveira Goulart} \\ Doutora em Ciências pela Universidade Federal do Rio Grande do Sul (UFRGS)
}

\section{Resumo:}

As Práticas Integrativas e Complementares (PICs) se inserem no modelo assistencial holístico estabelecendo o equilíbrio entre ciência, tecnologia e humanização, assim, promovem a melhoria da qualidade de vida dos usuários, podendo reduzir a farmaco-dependência. Este estudo teve como objetivo avaliar o consumo de medicamentos em indivíduos que utilizam PICs. Trata-se de um estudo de base populacional realizado no município de Rondonópolis, MT. A amostragem foi realizada em múltiplos estágios, estratificada e por conglomerados. Foram incluídos na pesquisa 177 indivíduos que utilizavam alguma PIC. A média de medicamentos consumidos por entrevistado foi usada como indicador da intensidade e as diferenças entre as médias de consumo foram comparadas por meio do teste de Kruskal Wallis. A prevalência de consumo de medicamentos entre usuários de PICs foi de 79,66\%. O consumo médio de medicamentos na população estudada foi de 2,13 (DP: 2,31), sendo significativamente superior em idosos, indivíduos que possuíam até 8 anos de estudo, que não trabalhavam, que buscaram o serviço de urgência no último ano e os que possuíam plano de saúde. Os medicamentos que atuam no sistema cardiovascular foram os mais consumidos. Os usuários de PICs apresentaram uma alta prevalência uso de medicamentos. É de grande importância que pesquisas e ações de educação em saúde visando a promoção de um consumo consciente de medicamentos sejam realizadas e implementadas entre os usuários de PICs.

Palavras-chave: Uso de Medicamentos, Terapias Complementares, Prevalência, Epidemiologia. 


\title{
Pharmacoterapeutical profile of users of complementary and integrative practices
}

\begin{abstract}
:
Complementary and Integrative Practices (CIPs) are part of the holistic care model. They establish a balance between science, technology and humanization, improve users' quality of life, and may reduce drug dependence. The aim was to evaluate drug consumption in individuals using CIPs. This was a population-based study conducted in the municipality of Rondonópolis, MT. Sampling was performed in multiple stages, stratified and by clusters. In the study, were included 177 individuals that used a CIPs. The average number of medications consumed per interviewee was used as an indicator of the intensity and the differences between the averages of consumption were compared through the Kruskal Wallis test. The prevalence of medication use among users of CIPs was $79.66 \%$. The average consumption of medications in the study population was 2.13 (SD: 2.31), it was significantly higher in elderly individuals, those with up to eight years of study, who did not work, who sought the emergency department in the last year and those who had health insurance. Medications that act in the cardiovascular system were the most consumed. Users of CIPs had a high prevalence of medication use. It is greatly important that research and health education actions aimed at the promotion of a conscious consumption of medicines are performed and implemented among users of CIPs.
\end{abstract}

Keywords: Drug Utilization, Complementary Therapies, Prevalence, Epidemiology.

\section{Perfil fármaco-terapéutico de los usuarios de las Medicinas Alternativas y Complementarias}

\section{Resumen:}

La práctica de las Medicinas Alternativas y Complementarias (MACs) es parte del modelo de atención integral que establece el equilibrio entre ciencia, tecnología y humanización, promoviendo la mejora de la calidad de vida de los usuarios y reduciendo la dependencia de drogas. El presente estudio tuvo como objetivo evaluar el uso de medicamentos en personas que hicieron uso de las MACs. El estudio, de base poblacional, ha sido realizado en la ciudad de Rondonópolis, MT. El muestreo se realizó en múltiples etapas, estratificadas y agrupadas. Un total de 177 individuos que usaron cualquier técnica de MAC se incluyeron en la investigación. El número promedio de drogas consumidas por encuestado se usó como un indicador de intensidad y las diferencias entre las medias poblacionales de consumo se compararon mediante la prueba de Kruskal-Wallis. La prevalencia del uso de medicamentos entre los que utilizaron al menos una MAC fue del 79,66\%. El consumo promedio de drogas en la población estudiada fue de 2,13 (DP: 2,31), siendo significativamente más alta en los ancianos, las personas que tenían hasta 8 años de escolaridad, las en situación de paro, las que buscaron el servicio de emergencia en el último año y aquellos que tenían seguro médico. Los fármacos que actúan en el sistema cardiovascular fueron los más consumidos. Nuestro estudio reveló que la prevalencia del uso de medicamentos por parte de los usuarios de las MAC es alta. Por lo tanto, es de gran importancia que la investigación en educación para la salud y las acciones destinadas a promover el consumo racional de medicamentos se lleven a cabo y se implementen entre los usuarios de las MAC.

Palabras clave: Utilización de Medicamentos, Terapias Complementarias, Prevalencia, Epidemiología.

\section{INTRODUÇÃO}

Com o crescimento do modelo biomédico, novas técnicas e inovações médicas reforçaram o conceito mecânico reducionista de atenção à saúde, ao valorizar o conhecimento 
técnico científico, priorizar a atuação intervencionista, a medicalização e atenção fragmentada do indivíduo. Após muitos anos de predominância da medicina alopática e deste modelo mecanicista como ferramenta terapêutica, profissionais e pacientes vêm sentindo cada vez mais a necessidade de um modo de tratamento que envolva a integralidade do ser e o conceba de forma holística. Assim, as Práticas Integrativas e Complementares (PICs) se inserem no modelo assistencial holístico, estabelecendo o equilíbrio entre ciência, tecnologia e humanização (LUZ, 2005; QUEIROZ, 2000).

O termo PICs é uma denominação brasileira para o que a Organização Mundial de Saúde define como sendo "Medicina Tradicional e Complementar", pois buscam incitar mecanismos naturais de prevenção e recuperação da saúde, sendo esta entendida como bemestar amplo, o que suscita uma interação complexa de fatores físicos, mentais, emocionais, sociais e espirituais (Brasil, 2017; SCLIAR, 2007). A proposta das PICs vem com a ideia de complementação e ampliação do acesso às ações de saúde na perspectiva da integralidade da atenção (ANDRADE e COSTA, 2010; BRASIL, 2006; OMS, 2013; THIAGO e TESSER, 2011).

No Brasil, a Política Nacional de Práticas Integrativas e Complementares no Sistema Único de Saúde (SUS) foi instituída com ênfase na atenção primária e envolve homeopatia, plantas medicinais, fitoterapia, medicina tradicional chinesa (práticas corporais e acupuntura), termalismo social, arteterapia, ayurveda, biodança, dança circular, meditação, musicoterapia, naturopatia, osteopatia, quiropraxia, reflexoterapia, reiki, shantala, terapia comunitária integrativa, yoga e a medicina antroposófica (BRASIL, 2017; OMS, 2013). De acordo com a Portaria no 971 de 3 de maio de 2006, as PICS são práticas que auxiliam os mecanismos naturais de cuidado de agravos e contribuem para a promoção da saúde e até mesmo inserção social dos que as utilizam (BRASIL, 2008; BRASIL, 2012; BRASIL, 2013; BRASIL, 2015).

Neste cenário, as PICs promovem a melhoria da qualidade de vida dos usuários, além de contribuir para o uso racional de medicamentos, podendo reduzir a farmaco-dependência do usuário (BRASIL, 2006; SAAD e MEDEIROS, 2009). Nos últimos anos, o uso de medicamentos tem sido constituído como uma verdadeira epidemia, o que pode estar associado a diversos fatores, tais como o aumento da prevalência de doenças crônicas não transmissíveis na população brasileira, a alta medicalização da saúde, o estímulo à prescrição de medicamentos, entre outros (SECOLI, 2010). O desafio atual é promover o consumo consciente de 
medicamentos e também incentivar o uso das práticas integrativas e complementares nos tratamentos de saúde. Neste contexto, esta pesquisa tem como objetivo avaliar o consumo de medicamentos em indivíduos que utilizam PICs em um município do sul de Mato Grosso.

\section{METODOLOGIA}

Foi realizado um estudo prospectivo, transversal de base populacional no município de Rondonópolis, MT. O presente estudo faz parte da pesquisa Práticas Integrativas e Complementares por Profissionais e Usuários do Sistema Único de Saúde em um Município do Estado de Mato Grosso, aprovada pelo comitê de ética em pesquisa da Universidade Federal de Mato Grosso CAAE: 74021417.8.0000.8088, sendo respeitados todos os aspectos éticos de pesquisa com seres humanos, de acordo com a Resolução n. 466/2012. Neste estudo foram considerados os indivíduos que informaram utilizar PICs.

Os participantes da pesquisa foram selecionados por meio de amostragem probabilística, estratificada, por conglomerados e obtida em dois estágios, sendo a unidade primária de amostragem o setor censitário e a secundária, o domicílio. Considerou-se prevalência desconhecida de 50\%, nível de confiança igual a 95\%, erro amostral de 5\%, e proporção esperada de 0,50. No primeiro estágio, foram sorteados 37 setores censitários com probabilidade proporcional ao tamanho - expresso pelo número de domicílios existentes em cada um deles segundo o Instituto Brasileiro de Geografia e Estatística (IBGE, 2010). No segundo estágio, uma amostra sistemática de domicílios foi sorteada em cada setor censitário, com base na listagem dos domicílios previamente arrolados. Nos domicílios com mais de um indivíduo presente no momento da coleta de dados foi realizado um sorteio com uso de uma tabela de números aleatórios.

Foram consideradas as PICs reconhecidas pelo Ministério da Saúde sendo elas: medicina tradicional chinesa/acupuntura, homeopatia, plantas medicinais, fitoterapia, termalismo social/crenoterapia, medicina antroposófica, arterapia, ayurveda, biodança, dança circular, meditação, musicoterapia, naturopatia, osteopatia, quiropraxia, reflexoterapia, reiki, shantala, terapia comunitária integrativa e yoga (BRASIL, 2017). 
Os dados foram coletados entre janeiro a abril de 2018, através de visitas domiciliárias. Foi utilizado um instrumento do tipo formulário semi-estruturado composto por questões referentes aos aspectos sociodemográficos, uso de serviços de saúde, consumo de medicamentos e utilização de PICs. Os participantes do estudo foram questionados acerca de todos os medicamentos consumidos nos últimos 15 dias. Para a validação do uso de medicamentos, utilizou-se a metodologia padrão descrita por Landry et, al. (1988), tal técnica consiste em solicitar aos participantes do estudo a embalagem, receita, bula ou blister de produtos farmacêuticos utilizados, visando evitar omissão, em geral por esquecimento de medicamentos em uso, e, além disso, para assegurar a veracidade das especialidades farmacêuticas informadas.

Os princípios ativos presentes em cada especialidade foram listados e organizados de acordo a classificação ATC (Anatomical Therapeutical Chemical), elaborada pelo Nordic Councilon Medicines e recomendada pela Drug Utilization Research Group da OMS para os estudos de utilização de medicamentos. Os medicamentos com mais de um princípio ativo ficaram classificados na classe terapêutica do principal componente, produtos com diferentes ações farmacológicas foram enquadrados levando-se em conta sua indicação terapêutica.

As variáveis estudadas foram idade, sexo, escolaridade, situação conjugal, cor autoreferida, trabalho, renda, uso de serviço de urgência no último ano, internação no último ano, uso da Estratégia de Saúde da Família (ESF) e plano de saúde.

Para a tabulação e análise dos dados, utilizou-se o programa Epi-Info versão 7.2.2.6. A média de medicamentos utilizados por entrevistado foi usada como indicador da intensidade. Ela foi calculada dividindo-se o número de medicamentos citado nas entrevistas pelo número de entrevistados. A variável resposta foi o número de medicamentos utilizados nos 15 dias anteriores à entrevista. As diferenças entre as médias foram comparadas por meio do teste de Kruskal Wallis. Para todos os testes, se utilizou nível de significância de 5\%.

\section{RESULTADOS}

Participaram do estudo 177 indivíduos. A tabela 1 mostra que a maioria da população estudada foi composta por homens (76,83\%), indivíduos na faixa etária de 18 a 59 anos 
(62,71\%) e que possuíam até 8 anos de estudo (53,67\%). Mais da metade da população possuía companheiro(a) (60,45\%), se autodeclararam branco $(69,49 \%)$, não trabalhava $(60,45 \%)$, tinham renda de até 2 salários mínimos (67,80\%), não frequentou o serviço de urgência $(62,15 \%)$ e nem estiveram internados $(84,18 \%)$ no último ano. A maior parte eram usuários da ESF $(75,71 \%)$ e não possuíam plano de saúde $(68,36 \%)$.

Tabela 1 - Características sociodemográficas e de uso de serviços de saúde de usuários de PICs. Rondonópolis, MT, 2018. (n=177).

\begin{tabular}{llrc}
\hline \multicolumn{1}{c}{ VARIÁVEIS } & $\mathbf{n}$ & $\mathbf{\%}$ \\
\hline Idade & & & \\
& $20-59$ anos & 111 & 62,71 \\
& $\geq 60$ mais & 66 & 37,29
\end{tabular}

Sexo

Feminino

Masculino

\section{Escolaridade}

Até 8 anos de estudo

95

82

41

136

23,17

76,83

Mais de 8 anos de estudo

\section{Situação Conjugal}

Sem companheiro

Com companheiro

Cor

Branca

Negra e outros

\section{Trabalho}

Sim

Não

\section{Renda}

Até 2 salários

Mais de 2 salários

\section{Serviço de urgência no último ano}

Sim

Não

\section{Internação no último ano}

Sim

Não

\section{Usa ESF}


Sim $134 \quad 75,71$

Não $\quad 43 \quad 24,29$

Possui plano de saúde

Sim $\quad 56 \quad 31,64$

ESF: Estratégia de Saúde da Família

Fonte: os autores.

As PICs mais utilizadas pela população estudada foram plantas medicinais $(67,68 \%)$, homeopatia (14,41\%) e medicamentos fitoterápicos (10,92\%). A tabela 2 descreve estes dados.

Tabela 2 - PICs utilizadas por indivíduos que consumiram medicamentos nos 15 dias anteriores a entrevista. Rondonópolis, MT, 2018.

\begin{tabular}{lcc}
\hline \multicolumn{1}{c}{ PRÁTICA INTEGRATIVA COMPLEMENTAR } & n & \% \\
\hline Plantas Medicinais & 155 & 67,68 \\
Homeopatia & 33 & 14,41 \\
Fitoterapia & 25 & 10,92 \\
Acupuntura & 9 & 3,93 \\
Yoga & 3 & 1,31 \\
Terapia Comunitária Integrativa & 2 & 0,87 \\
Termalismo & 1 & 0,44 \\
Meditação & 1 & 0,44 \\
Total & 229 & 100 \\
\hline
\end{tabular}

Fonte: os autores.

A prevalência do consumo de medicamentos entre usuários de PICs foi de 79,66\% ( $n=141)$. 0 consumo médio de medicamentos por indivíduos que realizam PICs foi de 2,13 (DP: $2,31)$, sendo significativamente superior $(p \leq 0,05)$ em idosos, indivíduos que possuíam até 8 anos de estudo, que não trabalhavam, que buscaram o serviço de urgência no último ano e os que possuíam plano de saúde (Tabela 3).

Tabela 3 - Consumo médio de medicamentos por usuários de PICs de acordo com as características sociodemográficas e uso dos serviços de saúde. Rondonópolis, MT, 2018.

\begin{tabular}{lcc}
\hline Variáveis & $\begin{array}{c}\text { Número médio de } \\
\text { medicamentos }\end{array}$ & Valor de \\
\hline Idade & 1,51 & $<0,001$ \\
$20-59$ anos & 3,18 &
\end{tabular}

Sexo

$\begin{array}{lll}\text { Feminino } & 1,59 & 0,12\end{array}$ 
Masculino

\section{Escolaridade}

Até 8 anos de estudo

Mais de 8 anos de estudo

\section{Situação Conjugal}

Sem companheiro

Com companheiro

Cor

Branca

Outros

1,45

1,23

Trabalho

Sim

Não

\section{Renda}

Até 2 salários mínimos

Mais de 2 salários mínimos

2,16

2,09

Serviço de urgência no último ano

Sim

Não

\section{Internado no último ano}

Sim

Não

\section{Usa ESF}

Sim

Não

\section{Possui plano de saúde}

Sim

Não

ESF: Estratégia de Saúde da Família

Fonte: os autores
1,28

2,69
, 19

2,46

2,07

2,09

2,28

2,78

$<0,001$

0,81

$<0,001$

0,49

0,05

0,13

0,36

0,002

Os medicamentos mais consumidos por usuários de PICs foram aqueles que atuam no sistema cardiovascular (33,93\%), sistema nervoso central $(29,91 \%)$ e trato alimentar e metabolismo (19,64\%) (tabela 4). Os fármacos mais utilizados foram losartana $(21,30 \%)$, hidroclorotiazida $(12,43 \%)$ e atenolol $(8,88 \%)$. 
Tabela 4 - Classes medicamentosas utilizadas por usuários de PICs, de acordo com a classificação ATC. Rondonópolis, MT, 2018

\begin{tabular}{lcc}
\hline \multicolumn{1}{c}{ Classificação ATC nível 1 } & n & \% \\
\hline C (Sistema Cardiovascular) & 76 & 33,93 \\
N (Sistema Nervoso Central) & 67 & 29,91 \\
A (Trato Alimentar e Metabolismo) & 44 & 19,64 \\
B (Sangue e Sistema Hematológico) & 16 & 7,14 \\
G (Sistema Geniturinário e Hormônios Sexuais) & 9 & 4,02 \\
H (Preparações Hormonais) & 8 & 3,58 \\
J (Anti-infecciosos de Uso Sistêmico) & 2 & 0,89 \\
D (Dermatológicos) & 2 & 0,89 \\
Total & 224 & 100 \\
\hline
\end{tabular}

Fonte: os autores

\section{DISCUSSÃo}

As PICs representam uma forma de ampliar as práticas do cuidar, pois oferecem ao usuário a possibilidade de escolha do tratamento que julgar mais adequado a sua realidade 19 (PARANAGUÁ et al., 2009). No município de Rondonópolis, MT, os indivíduos que utilizam PICs foram em sua maioria homens, com idade entre 20 a 59 anos, com até 8 anos de estudo, de baixa renda, não inseridos no mercado de trabalho, que não estiveram internados no último ano e que utilizavam a ESF. Um estudo realizado em Belo Horizonte, MG, apontou que a população usuária de PICs foi composta predominantemente por mulheres, indivíduos com idade entre 19 e 28 anos, de cor parda, com 9 a 11 anos de estudos e que não possuíam emprego (BORGES et al., 2011). Nos Estados Unidos, o uso da PICs é maior entre os adultos, mulheres e aqueles com maiores níveis escolaridade e renda (BARNES et al., 2008). Na Austrália, dentre os usuários de PICS predominam os adultos jovens, mulheres, pessoas com maior escolaridade e renda, com emprego e plano de saúde privado (XUE et al., 2007). O perfil de utilização de PICs pode ser influenciado por fatores religiosos, culturais e socioeconômicos (ALMEIDA, ALMEIDA e GOLLNER, 2000; GÓMES, 2003; AZEVEDO et al., 2004). Além disso, Rosa, Câmara e Béria (2011) afirmam que o conhecimento profissional em relação à utilização das PICs é capaz de influenciar na decisão do usuário sobre sua aplicação, induzindo assim a intenção pessoal do comportamento para realização e adesão a estas práticas. 
As PICs mais utilizadas pela população estudada foram plantas medicinais, homeopatia e fitoterápicos. Subramanian e Midha (2016), em uma pesquisa multicêntrica, descreveram que ioga, massagem, meditação e fitoterapia foram as práticas mais prevalentes. Losso e Freitas (2017) verificaram que fitoterapia, acupuntura e homeopatia foram as PICs mais utilizadas pela população do Estado de Santa Catarina. Gontijo e Nunes (2017) descreveram em sua pesquisa que as PICs mais realizadas em Itumbiara e Panamá, GO e Arapor, MG foram acupuntura, fitoterapia e homeopatia. Atualmente, ainda existem dificuldades para realização de estudos sobre PICS no Brasil, pois o termo e seu significado ainda não são amplamente explanados no contexto da saúde pública. Há muitas limitações, como o conhecimento da população sobre as PICS e a baixa inclusão destas como fonte de estudo nos cursos da área da saúde (COUTO et al., 2018).

A prevalência de uso de medicamentos por usuários de PICs em Rondonópolis, MT foi de 79,66\%. Um inquérito nacional realizado na Colombia apontou que $40 \%$ da população que utiliza terapias complementares consumiu medicamentos prescritos no último ano (BARNES et al., 2008). O uso de PICs concomitante ao de medicamentos alopáticos, apesar de ser considerado inofensivo, pode mascarar ou mesmo distorcer os efeitos do tratamento medicamentoso convencional, sobretudo quando se trata de consumo de plantas medicinais e fitoterápicos. O potencial para a ocorrência de interações deve ser sempre observado, o que exige a necessidade de se adotar uma atitude criteriosa no uso de medicamentos e PICS, considerando que tanto os saberes nativos quanto os saberes científicos são pertinentes (ELIAS e ALVES, 2002; SOUSA et al., 2012).

O consumo médio de medicamentos na população estudada foi de 2,13. A Pesquisa Nacional sobre Acesso, Utilização e Promoção do Uso Racional de Medicamentos (PNAUM) indicou que o número médio de medicamentos consumidos pela população brasileira em 2015, foi de 2,4 sendo que a região Centro-Oeste obteve valor de 2,0 (LIMA et al., 2017). Um estudo de base populacional realizado em Campinas, SP demonstrou que o consumo médio de medicamentos foi de 1,81 entre os homens e de 1,96 entre as mulheres (COSTA et al., 2011). Pesquisas que avaliem o uso de fármacos entre usuários de PICs são raros, o que sugere a necessidade de se conhecer o perfil farmacoterapêutico desta população, contribuindo assim, com a adoção de medidas de saúde mais específicas para este público. 
Nossos resultados indicaram que o consumo médio de medicamentos foi significativamente superior entre os idosos. Resultado semelhante foi descrito por Costa e colaboradores (2011), os quais observaram um aumento neste consumo médio de acordo com a idade. $O$ envelhecimento é uma fase complexa com perda de funções, diminuição da autonomia e maior morbidade (OLSSON, RUNNAMO, ENGFELDT, 2011). Em razão disso, é esperado que as pessoas idosas utilizem múltiplos medicamentos para o controle dessas doenças e manutenção da qualidade de vida, sobretudo, como uma forma de amenizar situações comuns do envelhecimento (FLORES e BENEVEGNU, 2008; FARIAS e SANTOS, 2012).

Os indivíduos que possuíam até 8 anos de estudo utilizaram mais medicamentos que aqueles com maior escolaridade ( $<<0,001)$. Inquéritos farmacológicos brasileiros corroboram com estes resultados (VOSGUERAU et al., 2011; COSTA et al., 2017). A correlação entre baixa escolaridade e o consumo de medicamentos é preocupante, pois os esquemas de tratamentos muitas vezes podem ser comprometidos devido às dificuldades de interpretação e entendimento, influenciando na correta utilização ou na não utilização (COSTA et al., 2017). Neste sentido, é essencial que os profissionais de saúde promovam uma assistência farmacológica adequada, sobretudo a este grupo populacional.

A análise do uso de medicamentos entre usuários de PICS revelou um uso médio superior na população que havia utilizado o serviço de urgência no último ano. Prado et al., (2016) afirmam que o consumo de medicamentos em indivíduos que procuram serviços hospitalares, como os de urgência, está associado ao uso de medicamentos conforme prescrição.

$\mathrm{Na}$ presente pesquisa, a população que possuía plano de saúde consumiu mais medicamentos do que a população que não possuía plano. Arrais et al., (2005) verificaram que o consumo de medicamentos possuiu como um dos fatores determinantes a cobertura do plano de saúde. Vosguerau et al., (2011) afirmam que ser filiado a um plano privado, favorece o acesso a um número maior de consultas médicas e, por conseguinte, aquisição de um maior número de medicamentos. Prado et al., (2016) também apresentam resultados semelhantes sobre a influência do plano de saúde no consumo de medicamentos.

As classes medicamentosas mais consumidas nesta pesquisa foram dos fármacos que atuam no sistema cardiovascular, sistema nervoso central e no trato alimentar e metabolismo. Estes dados estão de acordo com a literatura (Costa et al., 2011; Cruzeta et al., 
2013; Vosguerau et al., 2011; Oliveira et al., 2018; Goulart et al., 2019). Dentre as principais causas do maior consumo de fármacos cardiovasculares está a ampliação das políticas de acesso a medicamentos como a gratuidade de tratamento para hipertensão, por exemplo, pelo Programa Farmácia Popular (SCHMIDT et al., 2011; TAVARES et al,. 2016). É de grande importância que estudos e ações de educação em saúde visando a promoção de um consumo consciente de medicamentos sejam realizadas e implementadas em todo âmbito de prescrição e dispensação medicamentosa, abrangendo desde as ESFs até os setores privados de saúde (OMS, 2009).

Uma das limitações deste estudo é que por ser uma pesquisa transversal não há garantias sobre a relação temporal entre as exposições e os desfechos. Cabe também considerar que o período recordatório utilizado poderá influenciar nos resultados, todavia, foi solicitado aos participantes que apresentassem as embalagens dos medicamentos a fim de se evitar possíveis esquecimentos e enganos.

\section{CONCLUSÃO}

O consumo médio de medicamentos em usuários de PICS foi de 2,13, com prevalência na população estudada de 79,66\%. Os idosos, indivíduos com até 8 anos de escolaridade, que não possuíam trabalho, que utilizaram o serviço de urgência no último ano e possuíam plano de saúde apresentaram maiores médias de consumo de medicamentos. Os fármacos que atuam no sistema cardiovascular foram os mais utilizados. Os dados evidenciam a necessidade de promoção do uso racional de medicamentos entre os usuários de PICs. 


\section{REFERÊNCIAS}

ALMEIDA, A.M. de; ALMEIDA, T.M. de; GOLLNER, A.M. Cirurgia espiritual: uma investigação. Revista da Associação Médica Brasileira, São Paulo, p.194-200, 2000. Disponível em: <http://www.scielo.br/scielo.php?script=sci_arttext\&pid=S0104-2302000000300002\&lng=en\&nrm=iso>. Acesso em 13 de agosto de 2018. http://dx.doi.org/10.1590/S0104-42302000000300002.

AZEVEDO, A.M. et al. O Uso da Medicina Alternativa e Complementar por Pacientes com Epilepsia: Risco ou Benefício?J Epilepsy Clin Neurophysiol, SÃo Paulo, p.201-204, 2004. Disponível em:<http://homeopatia.yolasite.com/resources/PLANTAS_MEDICINAIS/O\%20Uso\%20da\%20Medicina\%20Alte rnativa\%20e\%20Complementar\%20por.pdf>. Acesso em 12 de dezembro de 2018.

ARRAIS, P.S.D. et al. Prevalência e fatores determinantes do consumo de medicamentos no Município de Fortaleza, Ceará, Brasil. Caderno de Saúde Pública, Rio de Janeiro, p.1737-1746, 2005. Disponível em: http://www.scielo.br/scielo.php?script=sci_arttext\&pid=S0102-311X2005000600021\&lng=en\&nrm=iso>. Acesso em 12 de dezembro de 2018. http://dx.doi.org/10.1590/S0102-311X2005000600021.

ANDRADE, J.T.; COSTA, L..FA. Medicina Complementar no SUS: práticas integrativas sob a luz da Antropologia médica. Saúde Social, SÃo Paulo, p.497-508, 2010. Disponível em http://www.scielo.br/scielo.php?script=sci_arttext\&pid=S0104-12902010000300003\&lng=en\&nrm=iso. Acesso em 13 de agosto de 2019. http://dx.doi.org/10.1590/S0104-12902010000300003.

BARNES, P.M; BLOOM, B; NAHIM, R.L. National Center for Complementary and Integrative Health. Complementary and Alternative Medicine Use Among Adults and Children: National Health Statistics Report, United States, 2008. Disponível em: https://nccih.nih.gov/sites/nccam.nih.gov/files/news/camstats/2002/report.pdf. Acesso em 15 de Dezembro de 2018.

BORGES, M.R. et al. As práticas integrativas e complementares na atenção à saúde da mulher: uma estratégia de humanização da assistência no hospital Sofia Feldman. Reme - Revista Mineira de Enfermagem, Belo Horizonte, p.105-113, 2011. Disponívem em: http://bases.bireme.br/cgibin/wxislind.exe/iah/online/?IsisScript=iah/iah.xis\&src=google\&base=BDENF\&lang=p\&nextAction=lnk\&expr Search=20312\&indexSearch=ID. Acesso em 12 de Dezembro de 2018.

BRASIL. Ministério da Saúde. Glossário de definições legais. Agência Nacional de Vigilância Sanitária, 2006. Disponível em: http://portal.anvisa.gov.br/documents/281258/308532/Relatorio_5_anos_DEFINITIVO.pdf/857a85f9-ab6e4358-b67c-1fob81bee7d1. Acesso em 25 de Janeiro de 2020.

BRASIL. Ministério da Saúde. Secretaria de Atenção à Saúde. Departamento de Atenção Básica. Política Nacional de Práticas Integrativas e Complementares no SUS - PNPIC-SUS /Ministério da Saúde, Secret, aria de Atenção à Saúde, Departamento de Atenção Básica. - Brasília: Ministério da Saúde, 2006. 92 p. Disponível em: http://bvsms.saude.gov.br/bvs/publicacoes/pnpic.pdf. Acesso em 13 de agosto 2018.

BRASIL. Ministério da Saúde. Secretaria de Atenção à Saúde. Departamento de Atenção Básica. Política nacional de práticas integrativas e complementares no SUS: atitude de ampliação de acesso. 2. ed. Brasília: Ministério da Saúde, $2008 . \quad$ Disponível em: http://bvsms.saude.gov.br/bvs/publicacoes/politica_nacional_praticas_integrativas_complementares_2ed.pd f. Acesso em 09 de agosto e 2018.

BRASIL. Ministério da Saúde. Secretaria de Atenção à Saúde. Departamento de Atenção Básica. Política nacional de práticas integrativas e complementares no SUS: atitude de ampliação de acesso. 2. ed. Brasília: Ministério da $\begin{array}{lllll}\text { Saúde, } & 84 & \text { p. } & 2012 . & \text { Disponível em: }\end{array}$ $\underline{\text { http://bvsms.saude.gov.br/bvs/publicacoes/politica_nacional_praticas_integrativas_complementares_2ed.pd }}$ f. Acesso em 09 de agosto de 2018. 
BRASIL. Ministério da Saúde. Secretaria de Atenção à Saúde. Departamento de Atenção Básica. Política nacional de práticas integrativas e complementares no SUS: atitude de ampliação de acesso. 2. ed. Brasília: Ministério da
Saúde,
100
$\mathrm{p}$
2013.
Disponível
em:

http://bvsms.saude.gov.br/bvs/publicacoes/politica_nacional_praticas_integrativas_complementares_2ed.pd f. Acesso 09 de agosto de 2018.

BRASIL. Ministério da Saúde. Secretaria de Atenção à Saúde. Departamento de Atenção Básica. Política nacional de práticas integrativas e complementares no SUS: atitude de ampliação de acesso. 2. ed. Brasília: Ministério da
Saúde,
96
p.
2015.
Disponível
em:

http://bvsms.saude.gov.br/bvs/publicacoes/politica_nacional_praticas_integrativas_complementares_2ed.pd f. Acesso em 09 de agosto de 2018.

BRASIL. Ministério da Saúde. Política Nacional de Práticas Integrativas e Complementares: informe maio 2017. Ministério da Saúde, 2017. Disponível http://189.28.128.100/dab/docs/portaldab/documentos/prt_849_27_3_2017.pdf. Acesso em 09 de agosto de 2018.

BRASIL. Portaria nº 849. Inclui a Artet,erapia, Ayurveda, Biodança, Dança Circular, Meditação, Musicoterapia, , Osteopatia, Quiropraxia, Reflexoterapia, Reiki, Shantala, Terapia Comunitária Integrativa e Yoga à Política Nacional de Práticas Integrativas e Complementares., Ministério da Saúde 27 de março de 2017. Disponível em: http://bvsms.saude.gov.br/bvs/saudelegis/gm/2017/prt0849_28_03_2017.html. Acesso em 08 de agosto de 2018.

COSTA, K.S. et al. Utilização de medicamentos e fatores associados: um estudo de base populacional no Município de Campinas, São Paulo, Brasil. Caderno de Saúde Pública, Rio de Janeiro, p.649-658, 2011. Disponível em: http://www.scielo.br/scielo.php?script=sci_arttext\&pid=S0102311X2011000400004\&lng=en\&nrm=iso. Acesso em 14 de fevereiro de 2019. http://dx.doi.org/10.1590/S0102-311X2011000400004.

COSTA, C.M.F.N. et al. Use of medicines by patients of the primary health care of the Brazilian Unified Health System. Revista de Saúde Pública, [s.l.], v. 51, n. 2, p.1-11, 22 set. 2017. Universidade de Sao Paulo Sistema Integrado de Bibliotecas - SIBiUSP. Disponível em: https://www.revistas.usp.br/rsp/article/view/139774. Acesso em 14 de fevereiro de 2019. http://dx.doi.org/10.11606/s1518-8787.2017051007144

COUTO, A.G. et al. Vittalle - Revista de Ciências da Saúde, Rio Grande, p.56-62, 2018. Disponível em: file:///C:/Users/Usuario/Downloads/7448-23305-1-PB\%20(1).pdf. Acesso em 15 de fevereiro de 2019.

CRUZETA, A.P.S. et al. Fatores associados à compreensão da prescrição médica no Sistema Único de Saúde de um município do Sul do Brasil. Ciência \& Saúde Coletiva, Rio de Janeiro, p.3731-3737, 2013. Disponível em: http://www.scielo.br/scielo.php?script=sci_arttext\&pid=S1413-81232013001200029\&lng=en\&nrm=iso. Acesso em 15 de dezembro de 2018. http://dx.doi.org/10.1590/\$1413-81232013001200029.

ELIAS, M.C.; ALVES, E. Medicina não-convencional: prevalência em pacientes oncológicos. Revista Brasileira de Cancerologia, Rio de Janeiro, p.523-532, 2002. Epub Jan 05, 2017. Disponível em: http://www1.inca.gov.br/rbc/n_48/v04/pdf/artigo6.pdf. Acesso em 08 de abril de 2019. http://dx.doi.org/10.1590/1981-7746-solo0040.

FARIAS, R.G.; SANTOS, SMA.. Influência dos determinantes do envelhecimento ativo entre idosos mais idosos. Texto Contexto Enfermagem, Florianópolis, p.167-176, 2012. Disponível em: http://www.indexf.com/textocontexto/2012pdf/21-167.pdf. Acesso em 15 de agosto de 2019.

FLORES, V.B.; BENVEGNð, LA. Perfil de utilização de medicamentos em idosos da zona urbana de Santa Rosa, Rio Grande do Sul, Brasil. Caderno de Saúde Pública, Rio de Janeiro, p.1439-1446, 2008. Disponível em: http://www.scielo.br/pdf/csp/v24n6/24.pdf. Acesso em 15 de agosto de 2019. 
GÓMEZ, M.B.D. Medicina occidental y otras alternativas: ¿es posible su complementariedad? Reflexiones conceptuales. Caderno de Saúde Pública, Rio de Janeiro, p.635-643, 2003. Disponível em: http://www.scielo.br/scielo.php?script=sci_arttext\&pid=S0102-311X2003000200030\&lng=en\&nrm=iso. Acesso em 16 de agosto de 2019. http://dx.doi.org/10.1590/s0102-311X2003000200030.

GONTIJO, M.B.A.; NUNES, MF. Práticas integrativas e complementares: conhecimento e credibilidade de profissionais do serviço público de saúde. Trabalho, Educação e Saúde, [s.l.], v. 15, n. 1, p.301-320, 5 jan. 2017. FapUNIFESP (SciELO). Disponível em: http://www.scielo.br/scielo.php?script=sci_arttext\&pid=S198177462017000100301\&lng=en\&nrm=iso. Acesso em 23 de fevereiro de 2019. http://dx.doi.org/10.1590/19817746-solo0040.

GOULAR, LS. et al. Medication consumption in a Brazilian area covered by the Family Health Strategy: Prevalence and associated factors. Escola Anna Nery, Rio de Janeiro, p.1-7, 2019. Disponível em: http://www.scielo.br/scielo.php?script=sci_arttext\&pid=S1414-81452019000200225\&lng=en\&nrm=iso. Accesso em 16 de abril de 2019. http://dx.doi.org/10.1590/2177-9465-ean-2018-0228.

IBGE. Instituto Brasileiro de Geografia e Estatística. Disponível em http://cidades.ibge.gov.br/xtras/perfil.php?codmun=510760. Acesso em 10 de dezembro de 2017.

LANDRY, J.A. et, al. Validation of two methods of data collection of self-reported medicine among the elderly. Gerontologist; v.28, n.5, p.672-676, 1988. Disponível em: https://academic.oup.com/gerontologist/articleabstract/28/5/672/630966?redirectedFrom=fulltext. Acesso em 15 de agosto de 2019. https://doi.org/10.1093/geront/28.5.672

LIMA, M.G. et al. Indicators related to the rational use of medicines and its associated factors. Revista de Saúde Pública, [s.l.], v. 51, n. 2, p.1-9, 22 set. 2017. Universidade de Sao Paulo Sistema Integrado de Bibliotecas - SIBiUSP. Disponível em: https://www.scielosp.org/scielo.php?pid=S003489102017000300316\&script=sci_arttext. Acesso em 16 de agosto de 2019. http://dx.doi.org/10.11606/s1518$\underline{8787.2017051007137 .}$.

LOSSO, L.N.; FREITAS, S.F.T. Avaliação do grau da implantação das práticas integrativas e complementares na Atenção Básica em Santa Catarina, Brasil. Saude Debate, Rio de Janeiro, p.171-187, 2017. Disponível em: http://www.scielo.br/pdf/sdeb/v41nspe3/0103-1104-sdeb-41-spe3-0171.pdf. Acesso em 15 de agosto de 2019.

LUZ, M.T. Cultura Contemporânea e Medicinas Alternativas: Novos Paradigmas em Saúde no Fim do Século XX. Revista de Saúde Coletiva, Rio de Janeiro, p.145-176, 2005. Disponível em: http://www.scielo.br/pdf/physis/v15s0/v15s0a08.pdf. Acesso em 23 de fevereiro de 2019.

OLIVEIRA, V.C.; NEVES, J.O.R; HELENA, E.T.S. Polifarmácia e padrão de utilização de medicamentos em Pomerode, SC.Arquivos Catarinenses de Medicina, Florianópolis, p.124-136, 2018. Disponível em: http://www.acm.org.br/acm/seer/index.php/arquivos/article/view/328/257. Acesso em 15 de agosto de 2019.

OLSSON, I.N; RUNNAMO, R.; ENGFELDT, P. Medication quality and quality of life in the elderly, a cohort study. Health And Quality of Life Outcomes, [s.l.], v. 9, n. 1, p.1-9, 2011. Springer Nature. Disponível em: https://www.researchgate.net/publication/51770809_Medication_quality_and_quality_of_life_in_the_elderly _a_cohort_study. Acesso em 08 de abril de 2019. http://dx.doi.org/10.1186/1477-7525-9-95.

ORGANIZACIÓN MUNDIAL DE LA SALUD. Estrategia de la OMS sobre medicina tradicional 2014-2023. Ginebra: Organización Mundial de la Salud; 75 p. 2013. Disponível em: https://apps.who.int/iris/bitstream/handle/10665/95008/9789243506098_spa.pdf?sequence=1\&isAllowed=y Acesso em 15 de janeiro de 2019. 
PARANAGUÁ, T.T.B.; BEZERRA, A.L.Q. Atuação do enfermeiro em um hospital especializado em práticas integrativas. Revista de Enfermagem Uerj, Rio de Janeiro, p.261-267, 2008. Disponível em: http://www.facenf.uerj.br/v16n2/v16n2a20.pdf. Acesso em 08 de abril de 2019. .

PEREIRA, K.G. et al. Polifarmácia em idosos: um estudo de base populacional. Revista Brasileira de Epidemiologia, [s.l.], v. 20, n. 2, p.335-344, jun. 2017. FapUNIFESP (SciELO). Disponível em: http://www.scielo.br/scielo.php?script=sci_arttext\&pid=S1415-790X2017000200335\&lng=en\&nrm=iso. Acesso em 11 de fevereiro de 2019. http://dx.doi.org/10.1590/1980-5497201700020013.

PRADO, M.A.M.B. do et al. Uso de medicamentos prescritos e automedicação em homens. Revista Brasileira de Epidemiologia, [s.l.], v. 19, n. 3, p.594-608, set. 2016. FapUNIFESP (SciELO). Disponível em: http://www.scielo.br/scielo.php?script=sci_arttext\&pid=S1415-790X2016000300594\&lng=en\&nrm=iso. Acesso em 08 de abril de 2019. http://dx.doi.org/10.1590/1980-5497201600030010.

QUEIROZ, M.S. O itinerário rumo às medicinas alternativas: uma análise em representações sociais de profissionais da saúde. Caderno de Saúde Pública, Rio de Janeiro, p.363-375, 2000. Disponível em: http://www.scielo.br/scielo.php?script=sci_arttext\&pid=S0102-311X2000000200007\&lng=en\&nrm=iso. Acesso em 20 de dezembro de 2018. http://dx.doi.org/10.1590/S0102-311X2000000200007.

ROSA, C.; CÂMARA, S.G.; BÉRIA, J.U. Representaç̃̃es e intenção de uso da fitoterapia na atenção básica à saúde. Ciência \& Saúde Coletiva, Rio de Janeiro, p.311-318, 2011. Disponível em: http://www.scielo.br/scielo.php?script=sci_arttext\&pid=S141381232011000100033\&lng=en\&nrm=iso. Acesso em 20 de dezembro de 2018. http://dx.doi.org/10.1590/S1413-81232011000100033.

SAAD, M; MEDEIROS, R. Terapias Complementares-Cuidados para Evitar Cuidados Adversos. Einsten: Educação Continuada em Saúde, São Paulo, p.42-3, 2009. Acesso em 20 de dezembro de 2018.

SANTOS, T.R.A. et al. Consumo de medicamentos por idosos, Goiânia, Brasil. Revista Saúde Pública, São Paulo, p.94-103, 2013. Disponível em: http://www.scielo.br/pdf/rsp/v47n1/13.pdf. Acesso em 8 de abril de 2019.

SCLIAR, M. HISTÓRIA DO CONCEITO SAÚDE. Revista de Saúde Coletiva, Rio de Janeiro, p.29-41, 15 mar. 2007. Disponível em: http://www.scielo.br/pdf/physis/v17n1/v17n1a03.pdf. Acesso em 20 de janeiro de 2019.

SCHMIDT, M.I. et al. Chronic non-communicable diseases in Brazil: burden and current challenges. The Lancet, [s.l.], v. 377, n. 9781, p.1949-1961, jun. 2011. Elsevier BV. Disponível em: https://www.ncbi.nlm.nih.gov/pubmed/21561658. Acesso em 27 de maio de 2019. $\underline{\text { http://dx.doi.org/10.1016/s0140-6736(11)60135-9 }}$

SECOLI, S.R. Polifarmácia: interações e reações polifarmácia: interações e reações adversas no uso de medicamentos por idosos. Revista Brasileira de Enfermagem, Brasília, p.136-140, 2010. Disponível em: http://www.scielo.br/scielo.php?script=sci_arttext\&pid=S0034-71672010000100023\&lng=en\&nrm=iso. Acesso em 11 de fevereiro de 2019. http://dx.doi.org/10.1590/\$0034-71672010000100023.

SOUSA, I.M.C. et al. Práticas integrativas e complementares: oferta e produção de atendimentos no SUS e em municípios selecionados. Caderno de Saúde Pública, Rio de Janeiro, p.2143-2154, 2012. Disponível em: http://www.scielo.br/scielo.php?script=sci_arttext\&pid=S0102-311X2012001100014\&lng=en\&nrm=iso. Acesso em 20 de dezembro de 2018. http://dx.doi.org/10.1590/S0102-311X2012001100014.

SUBRAMANIAN, K; MIDHA, I. Prevalence and Perspectives of Complementary and Alternative Medicine among University Students in Atlanta, Newcastle upon Tyne, and New Delhi. Int Sch Res Notices. 2016: 9309534. 2016. Disponível

em: https://www.researchgate.net/publication/301676983_Prevalence_and_Perspectives_of_Complementary_an 
d_Alternative_Medicine_among_University_Students_in_Atlanta_Newcastle_upon_Tyne_and_New_Delhi. Acesso em 27 de maio de 2019.

TAVARES, N.U.L. et al. Free access to medicines for the treatment of chronic diseases in Brazil. Revista de Saúde Pública, [s.1.], v. 50, n. 2, p.1-10, 2016. FapUNIFESP (SciELO). Disponível em: http://www.scielo.br/scielo.php?script=sci_arttext\&pid=S0034-89102016000300313\&lng=en\&nrm=iso. Acesso em 27 de maio de 2019. http://dx.doi.org/10.1590/s1518-8787.2016050006118.

THIAGO, S.C.; TESSER, C.D. Percepção de médicos e enfermeiros da Estratégia de Saúde da Família sobre terapias complementares. Revista Saúde Pública, São Paulo, p.249-257, 2011. Disponível em: http://www.scielo.br/scielo.php?script=sci_arttext\&pid=S0034-89102011000200003\&lng=en\&nrm=iso. Acesso em 20 de dezembro de 2018. http://dx.doi.org/10.1590/S0034-89102011005000002.

VOSGERAU, M.Z.S. et al. Consumo de medicamentos entre adultos na área de abrangência de uma Unidade de Saúde da Família. Ciência \& Saúde Coletiva, Rio de Janeiro, p.1629-1638, 2011. Disponível em: http://www.scielo.br/scielo.php?script=sci_arttext\&pid=S1413-81232011000700099\&lng=en\&nrm=iso. Acesso em 11 de fevereiro de 2019. http://dx.doi.org/10.1590/S1413-81232011000700099.

WORLD HEALTH ORGANIZATION. Report of the WHO Interregional Workshop on the Use of Traditional Medicine in Primary Health Care. Ulaanbaatar: World Health Organization; 2009. Disponível em: https://apps.who.int/medicinedocs/documents/s16202e/s16202e.pdf. Acesso em 20 de dezembro de 2018.

XUE C.CL.; ZHANG A.L; LIN V.; DA COSTA, C.; STORY D.F. Complementary and alternative medicine use in Australia: a national population-based survey. J Altern Complement Med, 13(6):643-650; 2007. Disponível em: https://www.liebertpub.com/doi/abs/10.1089/acm.2006.6355. Acesso em Acesso em 27 de maio de 2019.

(cc)) BY

Este trabalho está licenciado com uma Licença Creative Commons - Atribuição 4.0 Internacional. 